This item was submitted to Loughborough's Research Repository by the author.

Items in Figshare are protected by copyright, with all rights reserved, unless otherwise indicated.

\title{
Investigation of faster-RCNN inception Resnet V2 on offline Kanji handwriting characters
}

PLEASE CITE THE PUBLISHED VERSION

https://doi.org/10.1145/3415048.3416104

PUBLISHER

ACM

VERSION

AM (Accepted Manuscript)

\section{PUBLISHER STATEMENT}

(c) \{Owner/Author | ACM 2020. This is the author's version of the work. It is posted here for your personal use. Not for redistribution. The definitive Version of Record was published in PRIS 2020: 2020 International Conference on Pattern Recognition and Intelligent Systems, https://doi.org/10.1145/3415048.3416104

\section{LICENCE}

CC BY-NC-ND 4.0

\section{REPOSITORY RECORD}

Adole, Tony, Eran Edirisinghe, Baihua Li, and Chris Bearchell. 2020. "Investigation of Faster-rcnn Inception Resnet V2 on Offline Kanji Handwriting Characters". Loughborough University.

https://hdl.handle.net/2134/12965069.v1. 


\section{Investigation of Faster-RCNN Inception Resnet V2 on Offline Kanji Handwriting Characters}

\author{
Anthony Adole ${ }^{\dagger}$ \\ Department of Computer Science, \\ EPSRC Centre for Doctoral \\ Training in Embedded Intelligence \\ Loughborough University \\ United Kingdom \\ a.a.adole@lboro.ac.uk
}

\author{
Prof. Eran Edirisinghe and \\ Dr. Baihua Li \\ Department of Computer Science \\ Loughborough University \\ United Kingdom \\ E.A.Edirisinghe@lboro.ac.uk \\ B.li@lboro.ac.uk
}

\author{
Dr. Chris Bearchell \\ Surface Intelligence \\ Oxford, United Kingdom \\ chris- \\ bearchell@softwareimaging.com
}

\begin{abstract}
In recent years detection and recognition of Offline handwriting character has being a major task in the computer vision sector, researchers are looking at developing deep learning models to avoid the traditional approaches which involves the tedious task of using the conventional methods for feature extraction and localization. However, state-of-the-art object detection models rely upon region proposal algorithms as a result, they settle for object location principles, such network reduces the time period of those detection network, exposing region proposal computation as a bottleneck. Faster-RCNN is a popular model used for recognition purpose in many recognition tasks, the goal of this paper is to serve as a guide for Multi-Classification on offline Handwriting Document using Pre-trained Faster-RCNN with inception resnet v2 feature Extractor. The result obtained from the experiments shows improved pre-trained models can be used in solving the research question concerning handwriting detection and recognition.
\end{abstract}

\section{KEYWORDS}

Faster-RCNN inception v2, Deep Learning, Offline Handwriting, Kanji handwriting

\section{ACM Reference format:}

Anthony Adole, Prof. Eran Edirisinghe, Dr. Baihua Li, Dr. Chris Bearchell. 2020. Investigation of Faster-RCNN inception resnet v2 on offline kanji handwriting characters.

\section{Introduction}

Handwriting is a skill that is personal to individuals' and has distinct fundamental characteristics. It consists of artificial graphical mark which are written on a surface used for communication; its serves as a medium that helps in the transfer of information; this purpose is achieved by the graphical mark's which are conventionally related to a language [1]. The act of writing has been considered to have made communication and historical transfer of knowledge possible between cultures and civilization [1]. Each script has a set of symbols, which are known as characters or letters and they have certain basic and different shapes. There are rules for combining letters to represent higherlevel linguistic units. Handwriting is a skill developed by humans which they use for communication and transfer of information, due to advancement in the technological era there has been a lot of significant changes related to technology in terms of human and computer communication through handwriting. Handwriting character recognition is a technology widely used in this century; thou it still possesses some important challenges which are been studied. Handwriting character recognition has been one amongst the foremost fascinating and difficult research areas within the field of pattern recognition and image processing within the recent years [2]. In numerous applications between man and machine, it can improve the interface used in intelligent surface [2]. The history of existing algorithm used for handwriting recognition system are either offline or on-line, and the methodology in which the algorithms are being developed are either traditional machine learning or deep learning. However, using traditional machinelearning techniques requires careful engineering, significant domain experience and in-depth understanding to transform raw data into a feature vector that would be used to classify the input pattern whereas Sambit described deep learning techniques used in artificial Intelligence as a subset of the traditional machine learning that achieves great potential and flexibility by learning to represent data as a nested hierarchy of ideas, with every notion outlined in respect to less complicated ideas and a lot of abstract representations computed in terms of less abstract ones using its hidden layer (layer between the input and output layer) architecture in the neural network [3].In the offline recognition system, the handwritings are usually captured optically by a scanner and stored as an image. The nature of offline handwriting characters consists of several phases such as pre-processing of the handwriting character, Segmentation of the character or word segmentation, 
Feature extraction, Classifications and Recognition. Therefore, the input of each phase would be the output of the previous phase. The neural network in the offline system has successfully yield comparably high recognition accuracy level compared to the traditional machine learning techniques [4]. Therefore, this paper proposed the use of pre-trained Faster-RCNN Inception Resnet V2 model on kanji Handwriting character and on generated synthetic kanji handwriting document. The Novel contribution of the paper is the investigation into already developed multi-classification neural network model (Faster-RCNN Inception resnet V2) which can be used for character recognition and detection purposes. The second section talks about related works done using faster-rcnn model, while the third section explains the methodology of implementing this model and detailed explanation on the architecture in combining the model with this feature extractor. The fourth section would basically present the data collation processes and the result of the experiment while the fifth section would talk about the conclusion of the research.

\section{Related Work}

There has been recent success in text recognition and detection system with the use of convolution neural networks (CNN). The application of neural networks is found within classification, segmentation, recognition and scene text detection systems. Researchers like Batuhan and his colleagues used long short-term memory (LSTM) and CNN for an end to end framework for recognition and classification of characters on a document. Sutskever and his colleagues [5] conferred a general end to end approach to sequence learning that produces borderline assumption on the sequence structure. A multi-layered long short-term memory (LSTM) was presented and it was used to map the input sequence to a vector of a fixed dimensionality which lead to using another deep LSTM model to decode the target sequence from the vector. The algorithm was tested on the WMT-14 dataset (English to German dataset), and additionally the LSTM the not have difficulty in recognizing long sentences. Sutskever and his colleagues built up the LSTM algorithm to be able to learn sensible phrase and sentence representations that are sensitive to word order and are relatively invariant to the active and passive voice. They also found that the proposed LSTM performance improved when there is a reversal in the order of words from the source sentence which then makes the optimization problem easier [5]. The propose recognition model based on artificial neural network (ANN) by Simonyan and his colleagues was supported by novel feature extraction technique. The proposed system was implemented using MATLAB. The model accepts the input as a scanned image. Therefore, the input tends to undergo a sequence of pre-processing stages; binarization and normalization. However, features are extracted and matched from the stored data in the database. Using their proposed recognition system, they were able to achieve a good average recognition rate with minimum training time [6]. Oivind and his colleagues reviewed the best feature extraction methods which could be applied to offline recognition of segmented characters. They specified that the selection of a feature extraction method is probably the single most important factor in achieving high recognition performance in character recognition systems. However, different feature extraction models are designed for different representations of characters, for example the grey-level character image, binary character image, character contour, and character skeleton or character graph for individual character. The feature extraction methods are discussed in terms of invariance properties, expected distortions, constructability and variability of the characters. They discussed properties for each of the feature extraction methods and character representation forms before selecting specific feature extraction methods to be used on the total character recognition system [7].

\section{Methodology}

This section describes the Architecture of Faster-RCNN with inception resnet v2 model [8] from Google object detection API [9] and the experimental setup. However, an important aspect of every handwriting character detection on document requires Region of interest extraction algorithm. This would help in the extraction of areas containing handwriting characters. However, Faster-rcnn model was developed based on the convolutional neural network framework (CNN) [10]. The CNN framework has been effective for object detection and recognition task in images and video scene [10]. The CNN is made up of convolutional step, Rectified linear unit (Relu) activation function, Pooling and Fully connected layer [11]. In the convolutional step, features are extracted, and learning occurs using matrices of the inputted image while allowing cultivation of spatial relationship of each feature within the inputted image. Therefore, the output of this stage creates a feature map which allows the introduction of ReLu activation function that would replace all negative pixel values in the feature map by zero in order to insert non-linearity. In pooling, dimensionality is been reduced in the feature map. However, this step remains an important information for minimizing overfitting by reducing the number of parameters and computations in the network [12]. The final step is the fully connected layer [12], this step is a multi-layer perceptron which utilizes a SoftMax activation function in the output to ensure that the outputs are probabilities that add up to 1 . The aim of this layer is to utilize the features obtained and then use it to classify the input image into different classes depending upon the training data [12]. Faster R-CNN architecture is complex due to several moving parts in the model. Faster-RCNN is composed of 3 neural networks which are feature network, Region Proposal Network and a Detection network. Therefore, in the settings of Faster R-CNN detection happens in two stages. The first stage processes images using feature extractor networks (inceptionresnet v2) and features at some selected intermediate level (PreAuxLogits) are used to predict class-agnostic box proposals. The second stage shows the use of box proposals which are used to crop features from intermediate feature map obtained within the first stage. Therefore, the output of each stages are subsequently sent in as input to the feature extractor in order to predict a class and class-specific box refinement for each proposal. This model employs an Inception resnet V2 feature extractor for extracting 
useful feature maps [13]. This feature extractor combines the architecture of both inception and residual network to improve performance significantly. However, the Inception resnet V2 features extractor combines the optimization benefits conferred by residual connections with the computational efficiency of inception units [13]. The model is a pre-trained model released by Google and have been trained on the COCO dataset for public use. The Faster-RCNN Inception Resnet V2 API was built on top of Tensorflow, which is intended for constructing, training, and deploying object detection models. The reason for selection of this API is because of its past research on the support of both object detection and localization task as described in section 2. In addition, the model detects high number of objects per image as well as increased accuracy for object detection. However, it has been shown by Google Object detection API team that it has a relative speed of $620 \mathrm{~ms}$ and a mean accuracy precision $\left(\mathrm{mAP}\left[{ }^{\wedge} 1\right]\right)$ of 37. The Inception Resnet V2 feature extractor use batch normalization after convolutional layers [14]. The training of the model was done with asynchronous SGD with momentum optimizer value of 0.9 and initial learning rate was set to 0.0003 . However, the learning rate was then reduced to 0.003 after $700 \mathrm{k}$ steps. However, we extract features using inception resnet v2 from the "Mixed_6a" layer including its associated residual layer.

3.1. Network Setup. The model was trained using pre-trained network, that was trained on the COCO dataset [15]. Therefore, our custom dataset was trained using pre-trained weights obtained from the Faster_rcnn_inception_resnet_v2_atrous_coco [15] . The trained hyperparameters are the same as used for the original configuration file for the model. The experiment where performed using the framework of TensorFlow object detection API on a Linux machine with a Nvidia Ge-force GTX $1080 \mathrm{Ti}$ with a total dedicated memory of $11 \mathrm{~GB}$. However, we modified the configuration file for the model and the script in the TensorFlow object detection API in other to enable evaluation of all the images in the test directory.

\section{Experimental Results}

Before the experiment was performed, data augmentation steps were carried out on all the dataset (because it would help in increasing the accuracy and localization level). We performed two stages of experiment, the first stage of the experiment is based on a single handwriting character presented in a document and the second stage is based on synthetic document containing randomly selected kanji characters. The list of augmentation steps below were used on the images during the pre-processing stages of the dataset before it could be ready for training. Python programming was used to implement the augmentation steps on each of the images.

- Rotation of the characters in the image 30 degrees and 120 degrees due to human handwriting styles

- Addition of noise into the images containing the single handwriting character

- Application of blurring effect on each single handwriting character images

- $\quad$ Resize the images to the minimum scale of visibility
- Random scaling of the dataset

The dataset of the single handwriting kanji character was obtained from 49 individuals (Source: ETL Character Database)[16] where everyone wrote more than a thousand different characters but for the purpose of our evaluation of the model, we used 1000 different kanji characters. However, each augmentation steps where used as Pre-processing stages of the dataset, while the synthetic document (figure 1) was created from each single augmented dataset and then randomly placed onto an empty bitmap. Python Programming language was used for developing the code used for the CNN, and Google research model file which can be obtained from the GitHub link [15]was used to achieve the investigation of using this networks. In other for training to begin, $20 \%$ of the dataset was used as a testing set while $80 \%$ was used as training set [11]. Then to achieve a successful conversion to TensorFlow record file, a label map was created containing assigned ID Number for each Classes.

4.1 Training Setup and Result Obtained. After creation of the virtual environment using anaconda, the required packages listed was installed: TensorFlow, Tensor board, Matplotlib, Cython, Pillow, Pandas, OpenCV and NumPy. Afterwards, we linked our Virtual environment path to the downloaded Google Object detection research folder. This research folder provides access to the Object detection API which we used for our retraining. We then compile the protobuf files in other to create files with_pb2.py in the protos folder located in Object detection folder. We obtain our model configuration file from the sample folder located also in the object detection folder.We obtain our model configuration file from the sample folder located also in the object detection folder. When the above process has been verified by echoing the path, we then create our TensorFlow record file for training from $80 \%$ of our dataset and another Tensorflow record file with the rest $20 \%$ of the dataset which would be used for evaluating the model.

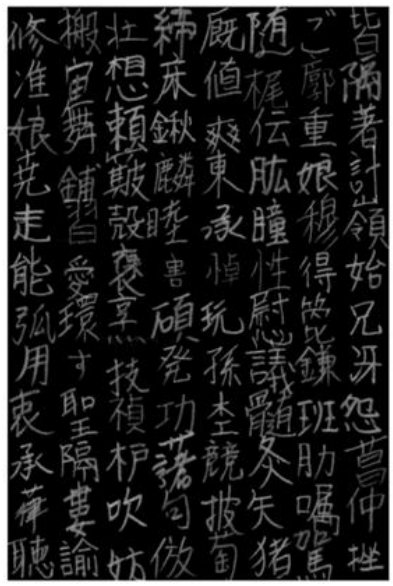

(a)

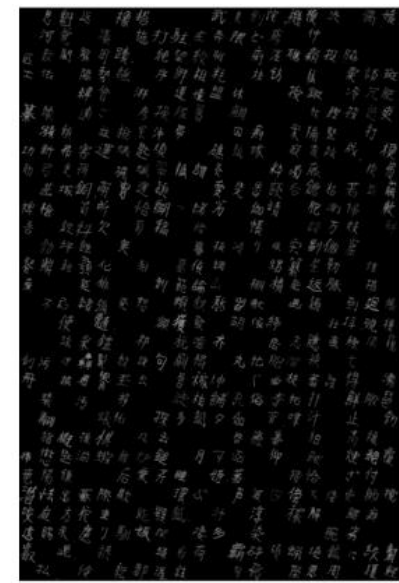

(b) 


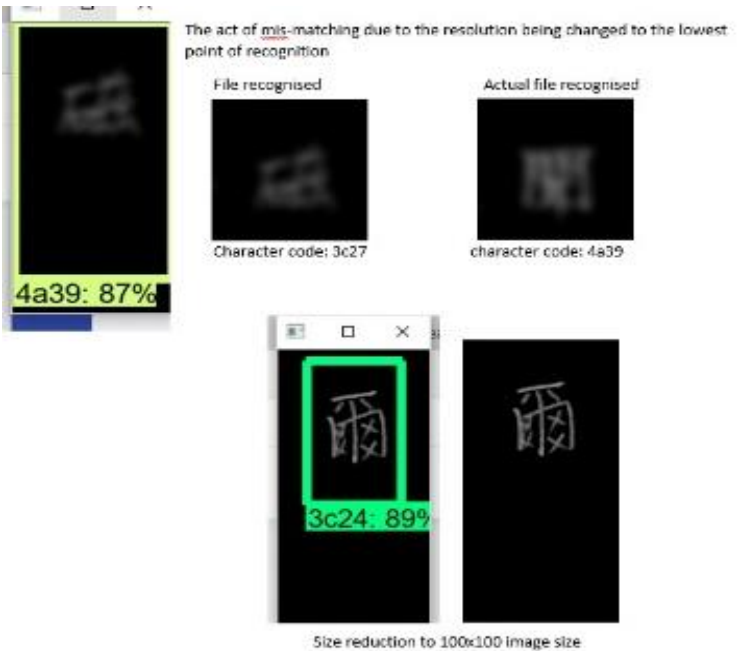

Figure 1: Sample of synthetic kanji handwriting document "a" been fully populated with characters while " $b$ " is spatially populated and contains slanted characters and A Case where the Image is resized to $100 \times 100$ and blurred.

However, we link our Tensorflow record files by applying the changes into the Faster-RCNN Inception resnet V2 configuration file. We also applied changes concerning our learning rate, how long should the training run, and the number of characters we want to identify with the model. After all the changes have been implemented, we start our training using the pre-trained FasterRCNN inception Resnet V2 model. The Training ran for 4 weeks due to the computational power of our machine which is described in network setup. Precision-Recall can be described as a useful measure of success for predicting when the classes are very imbalanced. Information retrieval precision is described as an evaluation of result relevancy, while recall can be described as an evaluation of how many relevant results are returned. Therefore, our mean accuracy precision obtained after the 4 weeks of the model been trained is $75.8 \%$.

For Faster R-CNN ResNet Inception V2 the initial Learning rate was started at 0.0003 and during the training it was change to 0.003 so that the module could learning quickly and help in the reduction of the Total loss. In other to check the performance of the models on images with poor resolution and size, I tested the model's frozen graph (which is 274-megabyte file size) with the test dataset. However, the model could perform better if enough training dataset of such situation is provided. Also, this specified test case is also difficult for human eyes. Observation made on this training results presented above are as follows:

- When the character is resized, it shows it can still be detected

- Mismatching of objects tend to occur when details of two object looks similar even with the naked eyes
Table 1: Graphical Presentation of Results during testing

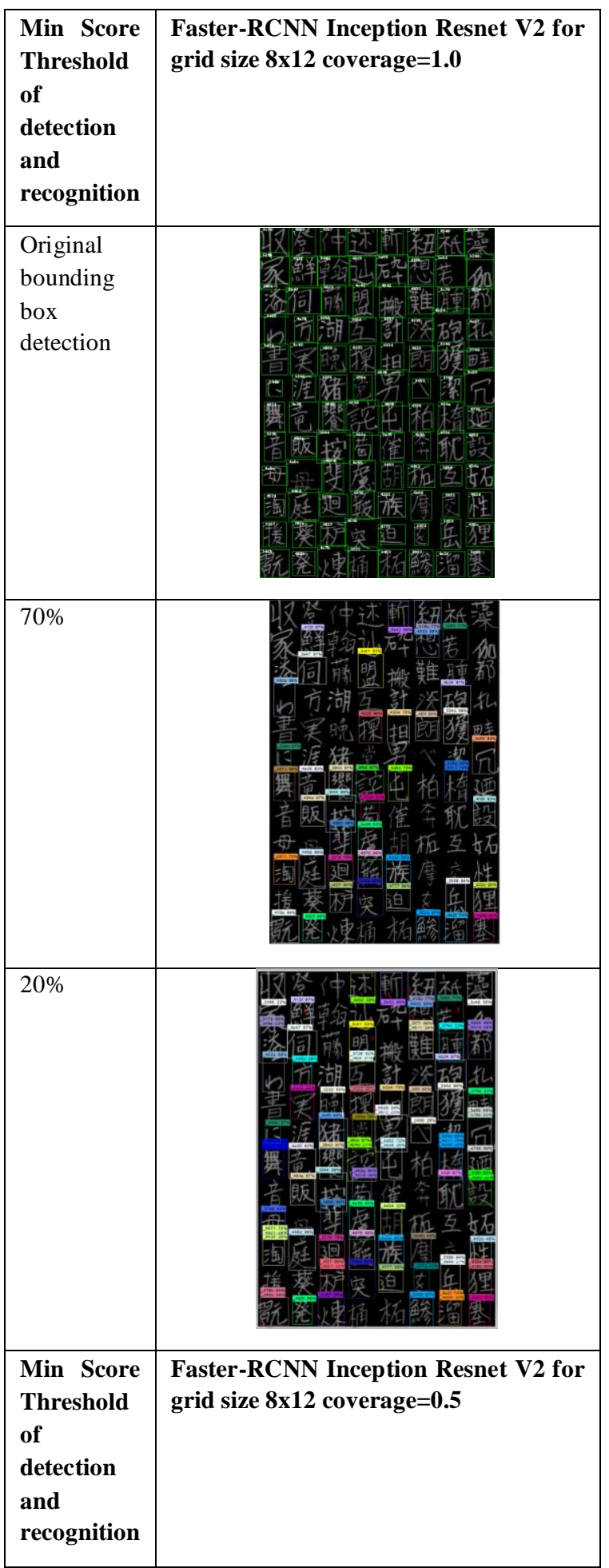




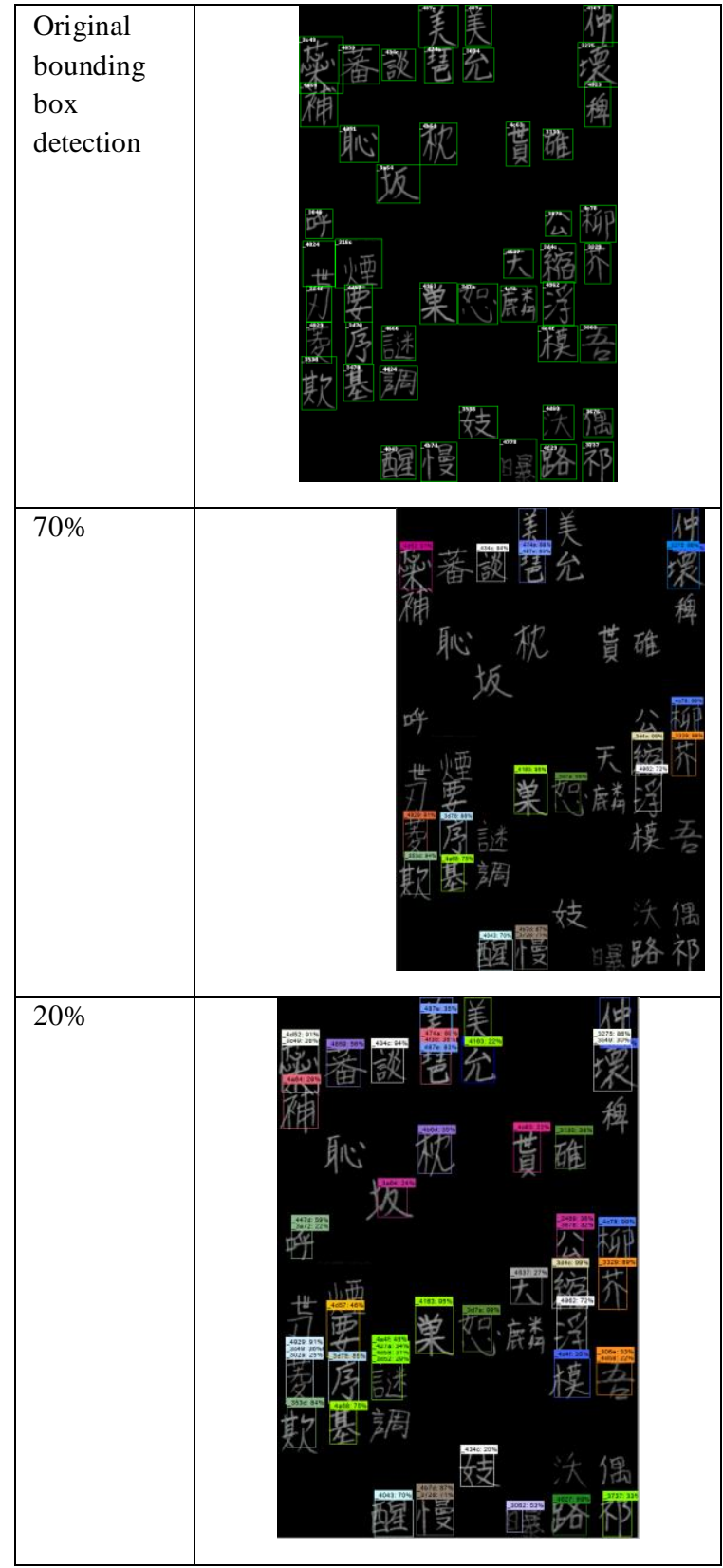

For the Synthetic dataset, the model was able to identify the characters located in every location on the image as shown in Table 1. However, with the mean accuracy precision at $75.8 \%$ the model could still identify every character in the images and predicting above $85 \%$ for each character identification and localization

\section{Conclusions}

Due to the results of the experiment presented (Table 1)., we hope that this detailed discussion will be a beneficial insight into various concepts involved in using pre-trained model and boost further advances in the area of handwriting and document detection and recognition. However, accurate recognition of the handwriting character on documents depends on the type of argumentation steps applied to the dataset, the type of dataset feed into the network and the type of feature extractor used. We used the pre-trained network and further trained it on our custom dataset which contains single scanned character and multiple characters on an image. We have observed that Faster-RCNN Inception Resnet V2 which we investigated could be used to Training more than 1000 classes without performing hierarchical classification of the classes. However, the module has its cons in respect to training of Highquality image dataset which has not been compressed or resized and cons for all characters located on the same location of all the dataset. We also explore the challenges in recognition of the Kanji Handwriting characters on synthetic dataset document. By investigating this model, we were able to present the performance of the model with how they perform with custom dataset, large classes and recognition accuracy on difficult and easily drawn offline Kanji Handwriting Characters. Though the training took a longer time for the model to converge due to computational power, it still was able to locate and identify correctly characters on a newly generated synthetic document.

\section{REFERENCES}

[1] R. Plamondon and S. N. Srihari, "On-line and off-line handwriting recognition: A comprehensive survey," IEEE Trans. Pattern Anal. Mach. Intell., vol. 22, no. 1, pp. 63-84, 2000

[2] S. Mori, C. Y. Suen, and K. Yamamoto, "Historical Review of OCR Research and Development," Proc. IEEE, vol. 80, no. 7, pp. 1029-1058, 1992.

[3] S. Mahapatra, "Towards Data Science," 2018. [Online]. Available: https://towardsdatascience.com/why-deep-learning-is-needed-over-traditionalmachine-learning-1b6a99177063. [Accessed: 08-Aug-2018].

[4] X. Y. Zhang, Y. Bengio, and C. L. Liu, "Online and offline handwritten Chinese character recognition: A comprehensive study and new benchmark," Pattern Recognit., vol. 61, pp. 348-360, 2017.

[5] I. Sutskever, O. Vinyals, and Q. V. Le, "Sequence to Sequence Learning with Neural Networks," pp. 1-9, 2014.

[6] M. Jaderberg, K. Simonyan, A. Vedaldi, and A. Zisserman, "Synthetic Data and Artificial Neural Networks for Natural Scene Text Recognition," pp. 1-10, 2014

[7] Ø. D. Trier, A. K. Jain, and T. Taxt, "Feature extraction methods for character recognition - A survey," Pattern Recognit., vol. 29, no. 4, pp. 641-662, 1996.

[8] J. Huang et al., "Speed/accuracy trade-offs for modern convolutional object detectors," in Proceedings - 30th IEEE Conference on Computer Vision and Pattern Recognition, CVPR 2017, 2017, vol. 2017-Janua, pp. 3296-3305.

[9] Nathan Silberman and Sergio Guadarrama, "TF-Slim: A high level library to define complex models in TensorFlow," Google AI, 2016. [Online]. Available: https://ai.googleblog.com/2016/08/tf-slim-high-level-library-to-define.html. [Accessed: 02-Dec-2018]

[10] S. Ren, K. He, R. Girshick, and J. Sun, "Faster r-cnn: Towards real-time object detection with region proposal networks." In Advances in neural information processing systems," IEEE Trans. Pattern Anal. Mach. Intell., vol. 39, no. 6, pp. 1137-1149, 2017.

[11] A. Krizhevsky, I. Sutskever, and G. E. Hinton., "Imagenet classification with deep convolutional neural networks. In Advances in neural information," $A d v$. Neural Inf. Process. Syst., pp. 1097-1105, 2012.

[12] Y. Lecun, Y. Bengio, and G. Hinton, "Deep learning," Nature, vol. 521, no. 7553, pp. 436-444, 2015.

[13] C. Szegedy et al., "Inception-v4, Inception-ResNet and the Impact of Residual Connections on Learning Christian," Popul. Health Manag., vol. 18, no. 3, pp. 186-191, 2014

[14] S. Ioffe and C. Szegedy, "Batch normalization: Accelerating deep network training by reducing internal covariate shift," in 32nd International Conference on Machine Learning, ICML 2015, 2015, vol. 1, pp. 448-456.

[15] Google API team, "Google Models and examples built with TensorFlow," GitHub, 2016. [Online]. Available: https://github.com/tensorflow/models [Accessed: 30-Apr-2018].

[16] etlcdb, "ETL Character Database," etlcdb, 2014. [Online]. Available: http://etlcdb.db.aist.go.jp/. [Accessed: 01-May-2018]. 